\title{
The Volumetric Rate of Calcium-rich Transients in the Local Universe
}

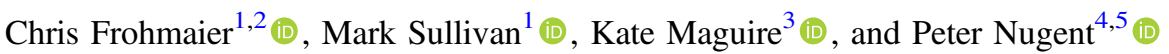 \\ ${ }^{1}$ Department of Physics and Astronomy, University of Southampton, Highfield, Southampton, SO17 1BJ, UK; m.sullivan@soton.ac.uk \\ ${ }^{2}$ Institute of Cosmology and Gravitation, University of Portsmouth, Portsmouth, PO1 3FX, UK; chris.frohmaier@port.ac.uk \\ ${ }^{3}$ School of Mathematics and Physics, Queen's University Belfast, Belfast BT7 1NN, UK \\ ${ }^{4}$ Department of Astronomy, University of California, Berkeley, CA, 94720-3411, USA \\ ${ }^{5}$ Lawrence Berkeley National Laboratory, Berkeley, CA, 94720, USA \\ Received 2018 February 13; revised 2018 March 30; accepted 2018 April 3; published 2018 May 4
}

\begin{abstract}
We present a measurement of the volumetric rate of "calcium-rich" optical transients in the local universe, using a sample of three events from the Palomar Transient Factory (PTF). This measurement builds on a detailed study of the PTF transient detection efficiencies and uses a Monte Carlo simulation of the PTF survey. We measure the volumetric rate of calcium-rich transients to be higher than previous estimates: $1.21_{-0.39}^{+1.13} \times$ $10^{-5}$ events $\mathrm{yr}^{-1} \mathrm{Mpc}^{-3}$. This is equivalent to $33 \%-94 \%$ of the local volumetric Type Ia supernova rate. This calcium-rich transient rate is sufficient to reproduce the observed calcium abundances in galaxy clusters, assuming an asymptotic calcium yield per calcium-rich event of $\sim 0.05 M_{\odot}$. We also study the PTF detection efficiency of these transients as a function of position within their candidate host galaxies. We confirm as a real physical effect previous results that suggest that calcium-rich transients prefer large physical offsets from their host galaxies.
\end{abstract}

Key words: galaxies: clusters: general - galaxies: clusters: intracluster medium - supernovae: general

\section{Introduction}

Over the past decade, all-sky optical time-domain surveys have uncovered a new class of so-called "Ca-rich" transients (Perets et al. 2010; Kasliwal et al. 2012; Valenti et al. 2014; Lunnan et al. 2017). These events have properties intermediate to those of supernovae ( $\mathrm{SNe}$ ) and novae, with characteristics that set them apart from the classical SN population: absolute magnitudes in the range of -15 to $-16.5 \mathrm{mag}$, a rapid photometric evolution with rise times of 10-15 days, photospheric velocities of $6000-11,000 \mathrm{~km} \mathrm{~s}^{-1}$, and a fast spectroscopic evolution to calcium-dominated nebular spectra.

The number of published events remains small: Lunnan et al. (2017) considered a combined sample of eight events with good photometric coverage. These objects all occurred in the outskirts of early-type galaxies, at $\sim 8-80 \mathrm{kpc}$ from the centers, and with a tentative preference for cluster environments. This suggests a metal-poor (and perhaps also old) explosion environment, ruling out a massive-star origin if they are formed in situ. Yuan et al. (2013) showed that their locations are consistent with globular cluster distributions. However, photometric searches for globular clusters at the positions of known Ca-rich transients have, on the whole, been unsuccessful (Lyman et al. 2014, 2016; Foley 2015; Lunnan et al. 2017).

Several progenitor scenarios have been suggested to explain the physical origin of Ca-rich events. These include heliumshell detonations (without core detonations) on the surface of low-mass carbon-oxygen (CO) white dwarfs (Waldman et al. 2011), collisions in binary systems involving an He donor and a CO or ONe white dwarf (García-Berro et al. 2017), and suggestions that they may result from the tidal disruption of $\mathrm{CO}$ white dwarfs by intermediate-mass black holes (Rosswog et al. 2009; Metzger 2012; Sell et al. 2015).

Ca-rich transients may also play an important role in other areas of astrophysics. A long-standing puzzle is the origin of the observed $\mathrm{Ca} / \mathrm{Fe}$ overabundance in the intracluster medium (ICM; de Plaa et al. 2007; Mernier et al. 2016a), which cannot be explained by the yields of traditional SN classes such as core-collapse SNe (CCSNe) and SNe Ia. Mulchaey et al. (2014) showed that a contribution from Ca-rich transients could provide a source of calcium to match the ICM measurements. The remote locations would also make them efficient at polluting the ICM, since their ejecta does not have to escape a galaxy potential (Zaritsky et al. 2004). The source of Galactic positrons has also been suggested to be due to faint thermonuclear $\mathrm{SNe}$ (Perets et al. 2010) that produce the positron emitter ${ }^{44} \mathrm{Ti}$, with the decay of ${ }^{44} \mathrm{Ti}$ leading to the production of ${ }^{44} \mathrm{Ca}$, either through the merger of two white dwarfs (Crocker et al. 2017) or as a natural by-product of He-shell detonation (Waldman et al. 2011).

However, the rate of Ca-rich transients is uncertain owing to the small numbers discovered to date. This is a by-product of the faintness and speed of their light curves, which make them difficult to detect. Absolute volumetric rates have not been calculated for a homogeneous sample of Ca-rich transients before; rather, rates relative to $\mathrm{SNe}$ Ia have been inferred. Perets et al. (2010) inspected the spectra of objects in the Lick Observatory Supernova Search (Filippenko et al. 2001) for possible Ca-rich candidates. An incompleteness assumption was made to produce a sample of $2.3 \mathrm{Ca}$-rich transients and 31 $\mathrm{SNe}$ Ia in the same search. They concluded that the Ca-rich rate is some 7\% of the SN Ia rate. Similarly, Kasliwal et al. (2012) placed a lower limit on the Ca-rich rate by comparing the number of Ca-rich events discovered in the Palomar Transient Factory (PTF; Rau et al. 2009) to the number of SNe Ia in the same volume. Without any assumption of PTF's incompleteness, they estimated that the Ca-rich transient rate must be $>2.3 \%$ of the SN Ia rate. A proper treatment of the survey incompleteness will also help explain the apparent preference for remote locations due to a potential for selection biases in their discovery (for example, it is easier to discover transients on fainter backgrounds away from galaxies).

The aim of this paper is to determine the absolute volumetric rate of Ca-rich transients, using a sample from PTF, and to determine whether they have a preference for the outskirts of galaxies. PTF was an automated optical sky survey operating at 

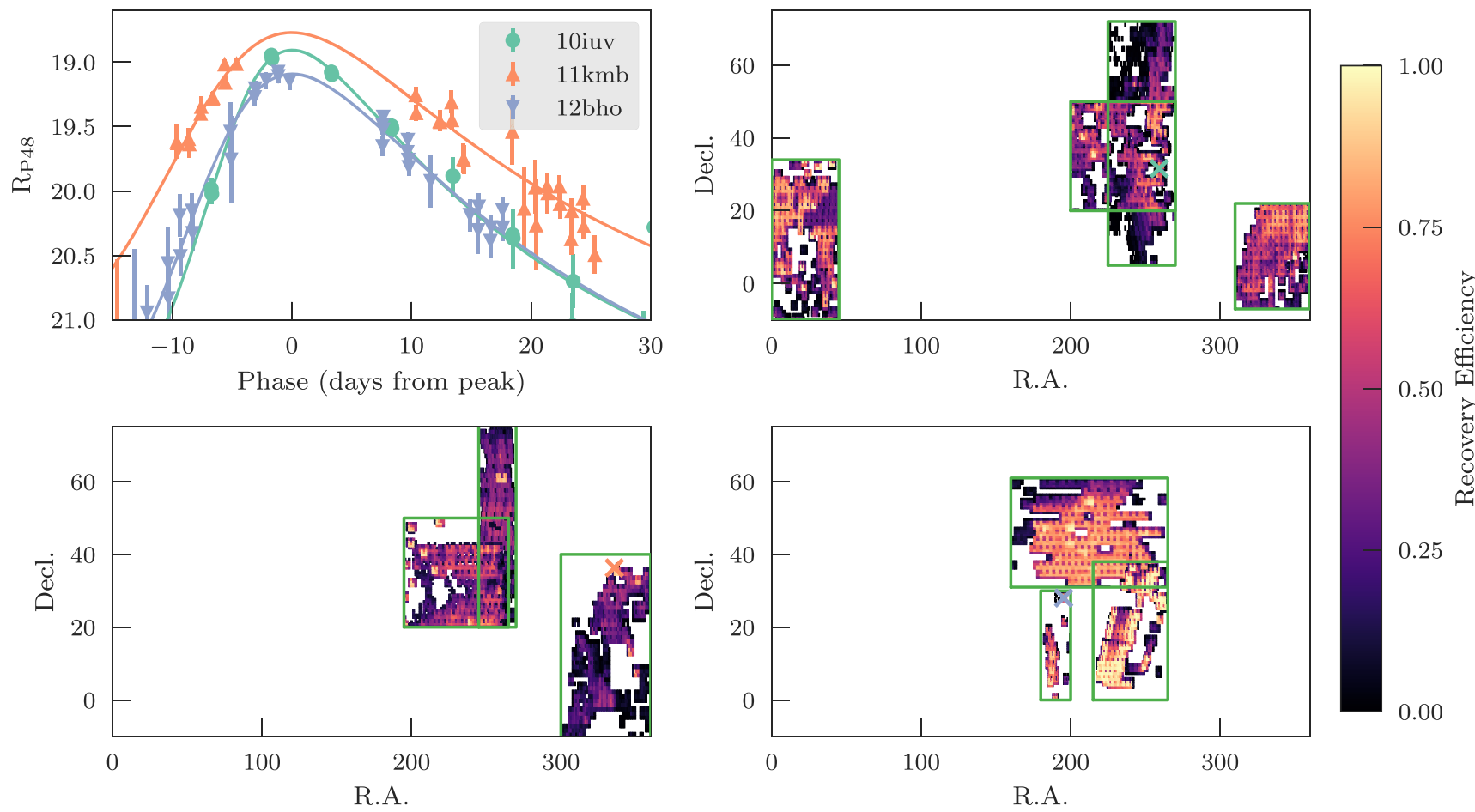

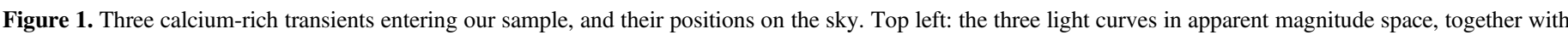

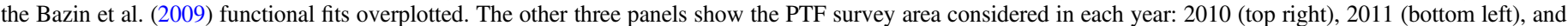

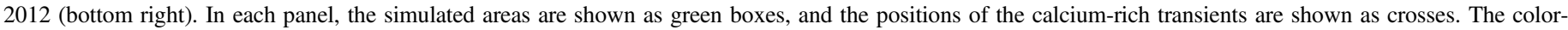
coding shows the fraction of simulated events that passed the coverage cuts.

the Samuel Oschin 48 inch telescope (P48) at the Palomar Observatory, specifically designed for transient detection. This paper builds on a comprehensive study of the PTF detection efficiencies presented in Frohmaier et al. (2017, hereafter F17) and applies them to a controlled sample of PTF Ca-rich events to estimate the first absolute rate of these events. Throughout, we assume a flat $\Lambda$ CDM cosmological model with $\Omega_{\mathrm{M}}=0.3$ and a Hubble constant of $H_{0}=70 \mathrm{~km} \mathrm{~s}^{-1} \mathrm{Mpc}^{-1}$.

\section{The Ca-rich Sample}

A parent sample of Ca-rich SNe in PTF was presented by Lunnan et al. (2017). This identified four likely members of the class (PTF11kmb and PTF12bho, along with PTF10iuv and PTF11bij previously identified by Kasliwal et al. 2012). Their sample also included PTF09dav (Sullivan et al. 2011). However, we have excluded this object, as it displays unusual photospheric spectra and late-time spectra that include hydrogen emission (Kasliwal et al. 2012), and thus it is likely not a member of the same class.

F17, and thus our study, is further restricted to the PTF survey period 2010-2012 (note that this would also exclude PTF09dav) and to periods when PTF was observing in the $R$-band filter $\left(R_{\mathrm{P} 48}\right)$, around $85 \%$ of the survey. We also require all objects in the sample to have been observed on at least four nights from -15 to +30 days from peak brightness, conventionally defined as day zero. PTF11bij was observed mainly in the PTF $g$-band filter and has insufficient $R_{\mathrm{P} 48}$ data to pass this requirement; thus, it is excluded from our analysis. Our final sample therefore has three events.

The light curves of these three events are shown in Figure 1, along with smoothed $R_{\mathrm{P} 48}$ light-curve templates fitted using the phenomenological functional form of Bazin et al. (2009), which is sufficient to fit the shape of most smoothly evolving
SNe. These model fits will be used in our simulations to model the Ca-rich events.

To determine the PTF spectroscopic completeness of Ca-rich transients, Lunnan et al. (2017) searched the PTF database for all objects that had luminosities between those of $\mathrm{SNe}$ and novae. They found one additional transient that may be a Ca-rich event (PTF10hcw), but its sparse light curve means that it does not pass our light-curve quality criteria. We note that if further objects were unconfirmed in the PTF database, this would have the effect of increasing the rate of Ca-rich transients that would be determined.

\section{The Rate of Ca-rich $\mathrm{SNe}$}

We next outline our method for determining the Ca-rich transient volumetric rate. The challenges are our small sample size, the large PTF observing footprint, and the variable cadence of the PTF observing strategy. We experimented with an "efficiency-based" method, weighting each event by its overall detection efficiency, as often used in high-redshift SN rate calculations from well-controlled surveys (e.g., Perrett et al. 2012). However, the small number of events in our sample, as well as the highly variable and sky-positiondependent detection efficiencies of our events, made this approach unreliable.

Instead, we used a Monte Carlo technique similar in concept to that of Prajs et al. (2017). Given an input intrinsic volumetric rate of events $\left(r_{\text {input }}\right)$, we simulate how many events PTF would have expected to detect based on its observing strategy $\left(N_{\mathrm{obj}}\right)$. By varying this input volumetric rate over a wide range of values and determining how often $N_{\text {obj }}=3$ in the simulations, we can construct the probability distribution of the volumetric Ca-rich rate, $r_{V}$. 
Key to this approach is the PTF detection efficiency study of F17. This study simulated around 7 million point sources in the PTF survey and studied their detection efficiency by the PTF pipeline as a function of source magnitude, host galaxy surface brightness, and various observing conditions (seeing or image quality, sky brightness, and limiting magnitude). From this, multidimensional detection efficiency grids were constructed, allowing the detection efficiency of any point source in any PTF image (and on any arbitrary host background) to be calculated.

Our detailed procedure is as follows. We first set the area and time period over which PTF would have been sensitive to Carich events. We chose 10 observational footprints from PTF over 2010-2012, with a total of $9428 \mathrm{deg}^{2}$ of sky. Each field was observed in a rolling cadence by PTF for between 30 and 155 days $\left(T_{i}^{\text {obs }} ; i\right.$ denotes the field, from 1 to 10$)$. These fields each have a volume $V_{i}$ over the redshift range $0.0035 \leqslant z \leqslant 0.03$, inside which PTF was sensitive to calcium-rich events. This volume is determined at the lower end by the redshift at which the calcium-rich transients would saturate the PTF detector $(z=0.0035)$ and at the upper end by the redshift at which the faintest of the calcium-rich transients would no longer be detected by PTF $(z=0.03)$.

A value for $r_{\text {input }}$ is randomly chosen from the interval $1 \times$ $10^{-6}$ events $\mathrm{yr}^{-1} \mathrm{Mpc}^{-3} \leqslant r_{\text {input }} \leqslant 5 \times 10^{-5}$ events $\mathrm{yr}^{-1} \mathrm{Mpc}^{-3}$ and used to randomly draw a number of events, $N_{\text {input }}$, from a Poisson distribution

$$
P\left(N_{\text {input }} ; \lambda\right)=\frac{\lambda^{N_{\text {input }}} e^{-\lambda}}{N_{\text {input }} !},
$$

where $\lambda=r_{\text {input }} \sum T_{i}^{\mathrm{obs}} V_{i}$ and the sum runs over the 10 fields. Each of these $N_{\text {input }}$ events is then randomly assigned a sky position, date of peak brightness, and redshift, such that they were uniformly distributed through the simulated volume and survey duration. Each event was then randomly assigned a Ca-rich light-curve template from Section 2, which was then scaled to the assigned redshift.

Simulations were then performed, replicating the operation of PTF and matching the nightly observing conditions and cadence patterns. Each time PTF "observed" an artificial Ca-rich transient, the F17 efficiencies were used to statistically assess whether PTF would have detected the event at that epoch. The recovered light curves of each simulated event were then checked to determine whether they met the light-curve quality criteria for the real sample: at least four detections over at least four nights. The number of "observed" Ca-rich events, $N_{\text {obj }}$, that met the criteria and the associated value of $r_{\text {input }}$ for that realization were recorded.

This process was repeated for $>1.7 \times 10^{6}$ realizations of the Ca-rich rate, at which point the statistical improvement from further simulations became negligible. This resulted in the analysis of $>3.5 \times 10^{8}$ simulated Ca-rich light curves. Clearly, this method of rate calculation is computationally expensive, but the results of the simulation are easy to understand and the uncertainties can be intuitively handled.

Figure 2 shows the fraction of our simulations that resulted in $N_{\text {obj }}=3$ as a function of $r_{\text {input }}$. The distribution is well described by a skewed Gaussian, and we use this functional form to estimate the most likely value of the Ca-rich rate and the region containing $68.3 \%$ of the probability. The volumetric

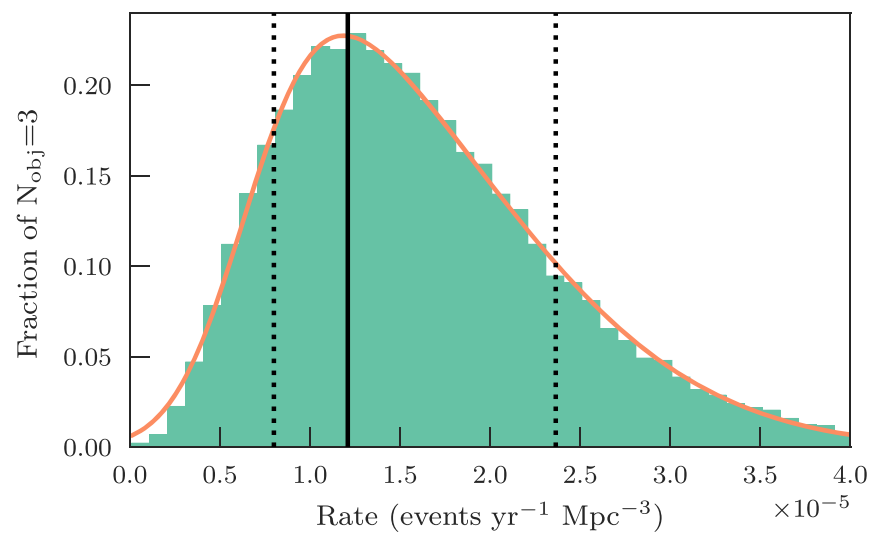

Figure 2. Fraction of our simulations that result in three Ca-rich transients being detected by PTF $\left(N_{\mathrm{obj}}=3\right)$ as a function of the input volumetric rate $r_{\text {input }}$. The overplotted function is a skewed Gaussian, with the solid vertical line denoting the median of the function and the vertical dotted lines denoting the $68.3 \%$ confidence interval. See Section 3 for details.

rate of Ca-rich transients is then

$$
r_{V}=1.21_{-0.39}^{+1.13} \times 10^{-5} \text { events } \mathrm{yr}^{-1} \mathrm{Mpc}^{-3} h_{70}^{3} .
$$

The volume-weighted mean redshift of the simulation was $z=0.023$.

This represents the first direct measurement of the Ca-rich transient rate and is equal to $\sim 50 \%$ of the SN Ia rate at a similar redshift (C. Frohmaier et al. 2018, in preparation). Combining our rate with assumptions on the performance of the Zwicky Transient Facility (ZTF; Bellm 2014; Laher et al. 2017), we estimate that ZTF should discover more than 20 Ca-rich transients each year. This order-of-magnitude improvement on the sample statistics will revolutionize our understanding of the volumetric rates, progenitors, and environments of these events.

\section{The Remote Locations of Calcium-rich Events}

We now consider the efficiencies of recovering a Ca-rich transient as a function of distance from its host galaxy. We use the real observations of each detected Ca-rich event's location and the survey performance around the object discovery date to assess whether each real Ca-rich event would have been discovered had it occurred in a different (but nearby) host environment. This allows at least a partial disentanglement of the intrinsic host environmental effects (e.g., bright galaxies) from observational effects (e.g., cadence and depth), in that we are restricted to survey periods and areas where the data were known to be good enough to discover Ca-rich events.

We begin by measuring the surface brightnesses for the Ca-rich transient host galaxies using high-quality reference images of the fields. The reference images were made from $R_{\mathrm{P} 48}$ observations, uncontaminated by $\mathrm{SN}$ light. Each image in the stack was resampled to a common coordinate system and co-added to produce a deep image. Potential host galaxies for our Ca-rich events were identified in the literature (Kasliwal et al. 2012; Lunnan et al. 2017) and form the sample for our study. We then measure these galaxies in the images using elliptical apertures and construct annuli containing fractions of the integrated galaxy light out to a distance of at least $45 \mathrm{kpc}$.

We then simulate each of the three Ca-rich template light curves from Figure 1 at a random position inside each of the elliptical annuli for each galaxy. The date of peak brightness 

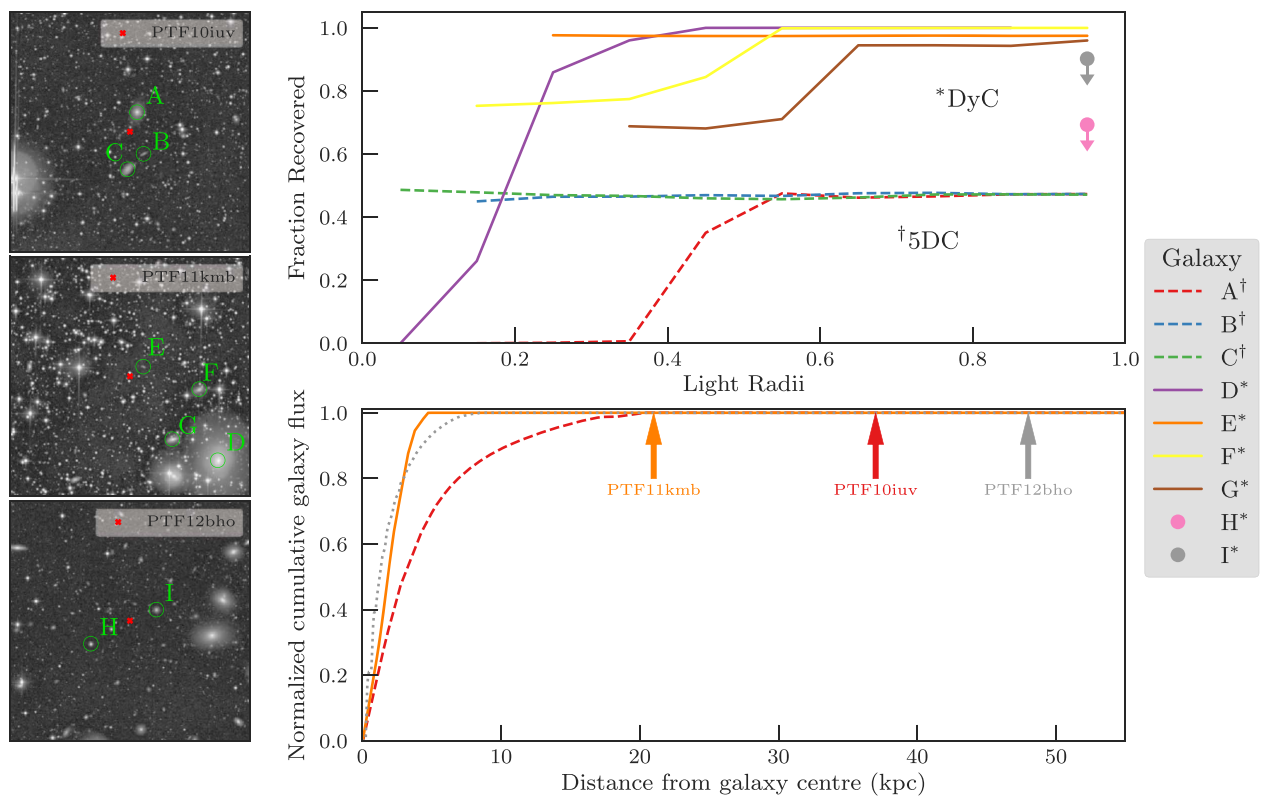

Figure 3. Study of the detection efficiency of the three PTF calcium-rich transients in our sample in relation to their host galaxies. Suspected host galaxies are identified in green, and their surface brightness profiles were measured. Ca-rich events were simulated in annuli containing fractions of galaxy light. In the top panel we show that if Ca-rich events followed the light profile, then PTF would have recovered more events than it missed. The efficiencies hit an apparent ceiling at larger radii as a result of the different PTF cadence experiments, called the "5-day-cadence" (5DC) or the "dynamic cadence" experiment (DyC). The median cadences of the PTF10iuv, PTF11kmb, and PTF12bho discovery fields were 4.95, 0.96, and 1.00 days, respectively. This result demonstrates that our Ca-rich sample is not significantly biased to remote locations, and these transients preferentially occur at large radii. The bottom panel shows the normalized cumulative fraction of galaxy light, as a function of distance from the galaxy core, for the nominal hosts. The arrows show the distance of each Ca-rich transient from the host and highlight the remote location of the explosion environment.

for each of the real Ca-rich transients loosely sets the date of our simulated events in the field: for each simulated event, we chose a random date of peak brightness to be within \pm 20 days of the real event's peak. We record the local surface brightness and semimajor axis distance to the center of the galaxy for each simulated event. We use the infrastructure of F17 to determine whether each simulated event would have been detected, again applying the requirement of four or more detections.

The results of our simulations - the fraction of events successfully recovered in each annulus-are shown in Figure 3. We find that galaxies with the brightest cores show a reduced recovery efficiency. However, under the assumption that the $R_{\mathrm{P} 48}$-light approximately traces the galaxy stellar mass, simulated Ca-rich events contained within the outer $\sim 50 \%$ of the stellar mass are recovered with a near-constant efficiency.

Furthermore, all the Ca-rich events in our sample were found at larger distances than an isophote containing $100 \%$ of the observable stellar light (i.e., where the galaxy flux becomes indistinguishable from the background).

The final two galaxies in Figure 3 ( $\mathrm{H}$ and I) had bright cores, such that most of the luminosity was contained in an area smaller than our spatial resolution. We therefore were unable to reliably split our simulations into different elliptical annuli, and instead we show the fraction of recovered events within a single boundary containing $90 \%$ of the luminosity/stellar mass.

These simulations demonstrate that if the radial distribution of Ca-rich transients follows the stellar mass of galaxies, PTF would have been capable of finding more events than it missed, i.e., the recovery fraction for the speculated hosts is $\sim 50 \%$ or more in an annulus containing 50\% of the stellar mass. Furthermore, we performed a simple Monte Carlo simulation of Ca-rich events under the assumption that they follow the host stellar light profile. We found that $<8 \times 10^{-5} \%$ of the simulations would produce all three events beyond the contours enclosing 99\% of the host light. This allows us to confidently reject any hypothesis that suggests that Ca-rich transients follow the stellar light. We conclude that the PTF Ca-rich transient sample is not significantly biased owing to the performance of the PTF experiment and that the large separation between event and host galaxy is an astrophysically real phenomenon. This confirms previous studies that show that Ca-rich transients do not follow a host stellar mass profile (Yuan et al. 2013), but with a robust consideration of the efficiency of the PTF experiment.

A final consideration on the detection efficiency relates back to the rates calculated in Section 3. If indeed there are Ca-rich transients in the cores of galaxies, where our results suggest that PTF has difficulty finding such events, then these objects would increase the absolute volumetric rate we find. Since we derive our Ca-rich rate from a sample of observed events, strictly speaking it should be considered as a lower limit if a significant population of Ca-rich events are later found to occur in galaxy cores.

\section{Intracluster Medium Calcium Abundances}

In this section we discuss the implications of our rate calculation with respect to the so-called "Calcium Conundrum." Observations of the ICM show a $\mathrm{Ca} / \mathrm{Fe}$ overabundance when expected elemental yields from both $\mathrm{SNe}$ Ia and CCSNe are included in the enrichment (e.g., de Plaa et al. 2007). It has been shown that a non-negligible contribution from Ca-rich $\mathrm{SNe}$ can potentially resolve this observed discrepancy (Mulchaey et al. 2014; Mernier et al. 2016b).

We use the latest ICM abundance measurements (Mernier et al. 2016a) of nine elements $\mathrm{X}=(\mathrm{O}, \mathrm{Ne}, \mathrm{Mg}, \mathrm{Si}, \mathrm{S}, \mathrm{Ar}, \mathrm{Ca}$, $\mathrm{Fe}$, and $\mathrm{Ni}$ ) from XMM-Newton observations of 44 galaxy 


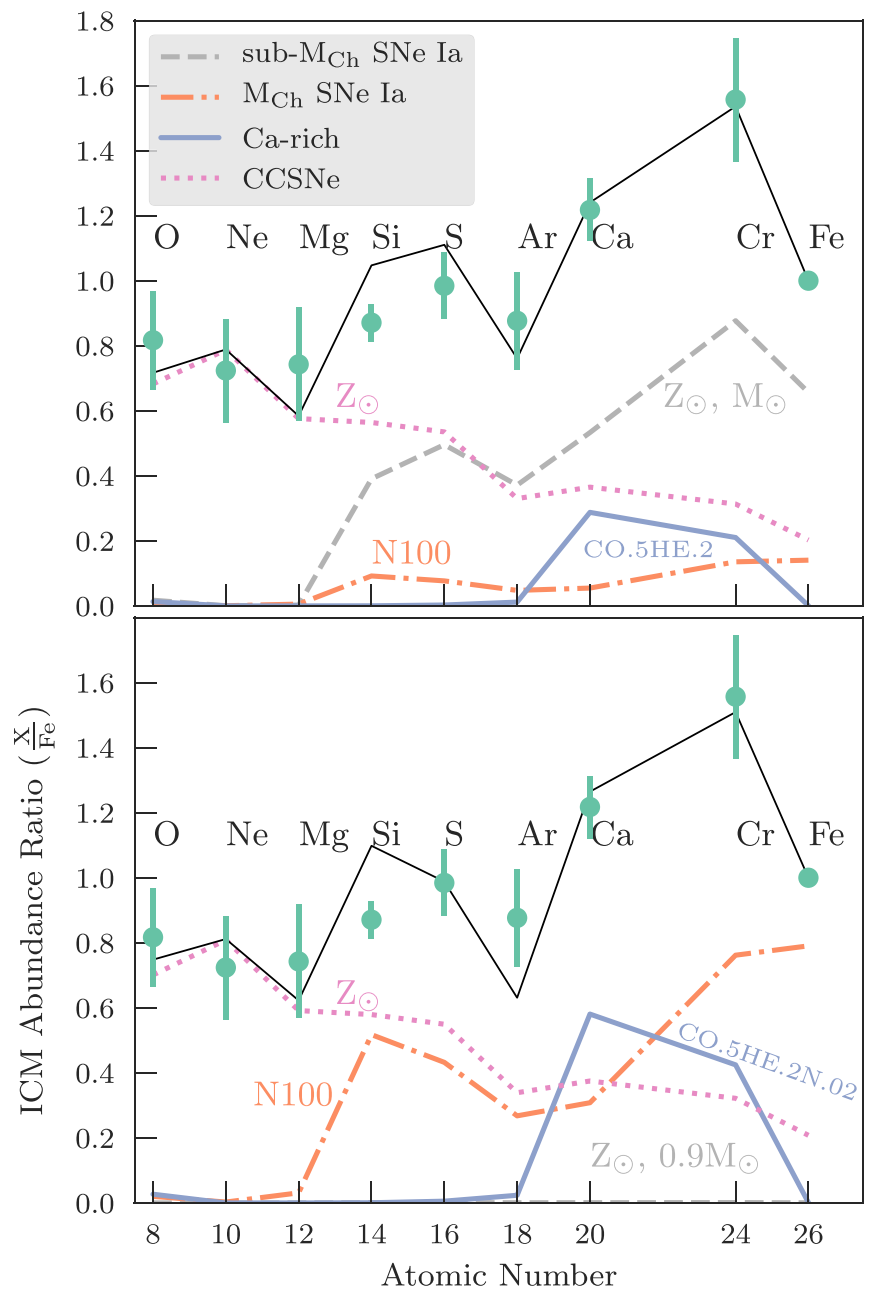

Figure 4. Observed ICM abundances from Mernier et al. (2016a) are shown by the green circles, and the relative $\mathrm{SN}$ contributions are shown by the various colored lines. The overall contribution from our SN models is shown by the solid black line. The top panel represents our best-fit combination of SNe and requires a dominant population of sub- $M_{\mathrm{Ch}} \mathrm{SNe}$ Ia. The next-best Ca-rich model is shown in the bottom panel and requires a negligible contribution from sub- $M_{\mathrm{Ch}} \mathrm{SNe}$ Ia. We find that the required Ca-rich rate to explain these abundances is highly dependent on initial metallicity/mass of our other SN classes, but that in all cases a significant $\mathrm{Ca}$-rich population is required.

clusters. We then build on the work of Mernier et al. (2016b), using their abunfit routine, to estimate the ratio of different SN classes that contribute to the ICM pollution. We do not attempt to definitively state the relative rates of the different SN types; rather, we investigate whether the contribution of $\mathrm{Ca}$-rich $\mathrm{SNe}$ is compatible with our rate result.

The asymptotic elemental mass yields for our SN Ia population are described by the Chandrasekhar-mass $\left(M_{\mathrm{Ch}}\right)$ N100 models of Seitenzahl et al. (2013) and the latest subChandrasekhar-mass (sub- $M_{\mathrm{Ch}}$ ) models of Shen et al. (2017). We select our sub- $M_{\mathrm{Ch}}$ models to have an initial metallicity of 0.5 or $1 Z_{\odot}$ and $\mathrm{C} / \mathrm{O}$ WD masses of 0.9 or $1.0 M_{\odot}-$ which best describe "normal" SNe Ia. The implementation of both the CCSNe (Nomoto et al. 2013) and the Ca-rich helium-shell detonation models (Waldman et al. 2011) into abunfit is described in Mernier et al. (2016b). The initial metallicities allowed for the CCSNe are $Z_{\text {int }}=(0,0.001,0.004,0.008,0.02$, $0.05)$, and a Salpeter initial mass function is assumed.
The abunfit routine performs a least-squares minimization to find the relative ratios of the $\mathrm{SN}$ classes that contribute to the $\mathrm{X} / \mathrm{Fe} \mathrm{ICM}$ abundances. The production of $\mathrm{Mn}$ and $\mathrm{Ni}$ is especially sensitive to the initial metallicity for the SNe Ia and the progenitor model used; furthermore, $\mathrm{Ni}$ is poorly constrained in the ICM abundance measurements. We therefore exclude $\mathrm{Mn}$ and $\mathrm{Ni}$ from our fits. In total, 336 model combinations were fit to the ICM observations, with 149 producing physically meaningful results. Results were rejected if they required nonpositive contributions from any model yield.

We find that our best-fit result $\left(\chi^{2} /\right.$ dof $\left.\sim 2.6\right)$ uses a combination of the CO.5HE. $2^{6}$ Ca-rich model, the $Z_{\odot}$ CCSN model, the N100 SN Ia model, and the $Z_{\odot}, 1 M_{\odot}$ sub- $M_{\mathrm{Ch}} \mathrm{SN}$ model. Our next best-fitting $\left(\chi^{2} /\right.$ dof $\left.~ 3.9\right)$ Ca-rich model, CO.5HE.2N.02, requires the same CCSN and $M_{\mathrm{Ch}}$ models as before, but a different sub- $M_{\mathrm{Ch}} \operatorname{model}\left(Z_{\odot}, 0.9 M_{\odot}\right)$.

The relative elemental yields for these results are shown in Figure 4 and clearly confirm previous claims (Mulchaey et al. 2014; Mernier et al. 2016b) that the ICM measurements require a non-negligible contribution from $\mathrm{Ca}$-rich $\mathrm{SNe}$ to explain the $\mathrm{Ca} / \mathrm{Fe}$ abundance ratio.

However, we note that while a contribution from a $\mathrm{Ca}$-rich model is required to produce good fits to the ICM, their relative contribution can vary significantly depending on the CCSN and SN Ia models we select. We demonstrate this by analyzing different CCSN and SN Ia model combinations for a fixed $\mathrm{Ca}$-rich model. We then calculate the ratio of $\mathrm{Ca}$-rich $\mathrm{SNe}$ to SNe Ia and scale the SN Ia rate evolution (Dilday et al. 2010; C. Frohmaier et al. 2018, in preparation) to estimate the Ca-rich rate. Our results are shown in Figure 5, where any fit utilizing the CO.5HE. 2 or CO.5HE.2N.02 model, with a $\chi^{2} /$ dof $<5$, is considered. These results place the $\mathrm{Ca}$-rich rate within the range of $19 \%-53 \%$ of the SN Ia rate. This is compatible with both our rate from Section 3 and the rate prediction of Mernier et al. (2016b). They found that Ca-rich SNe made up 34\% of their "thermonuclear" population, corresponding to a rate $\sim 51 \%$ of the SN Ia rate.

Finally, we note that directly comparing $\mathrm{SN}$ rates derived from ICM abundances and SN rates from direct observations is a limited approach. The ICM abundance SN rates only trace events that contribute to this environment, and not elemental yields trapped in a galaxy potential. This would not necessarily be a problem if the spatial distribution of all SN classes were identical. However, as we have demonstrated in Section 4, Ca-rich SNe preferentially occur at large distances from their host-making them more effective polluters of the ICM. The SN rates measured using the ICM abundance method are also very dependent on the elemental yields estimated from the various explosion models.

\section{Conclusion}

We have presented three results relating to Ca-rich faint-and-fast transients: (i) using detailed simulations of PTF, we have calculated the volumetric rate of Ca-rich transients to be 33\%-94\% of the SN Ia rate, higher than previously suggested; (ii) we confirm, using detection efficiencies from PTF, that Ca-rich events have an intrinsic preference for locations significantly offset from their host

\footnotetext{
6 The naming scheme for the Waldman et al. (2011) models represents the mass of the $\mathrm{C} / \mathrm{O} \mathrm{WD}$ core and masses of additional layers. For example, a $0.5 M_{\odot} \mathrm{C} / \mathrm{O}$ WD with a $0.2 M_{\odot}$ helium shell is listed as CO.5HE.2.
} 


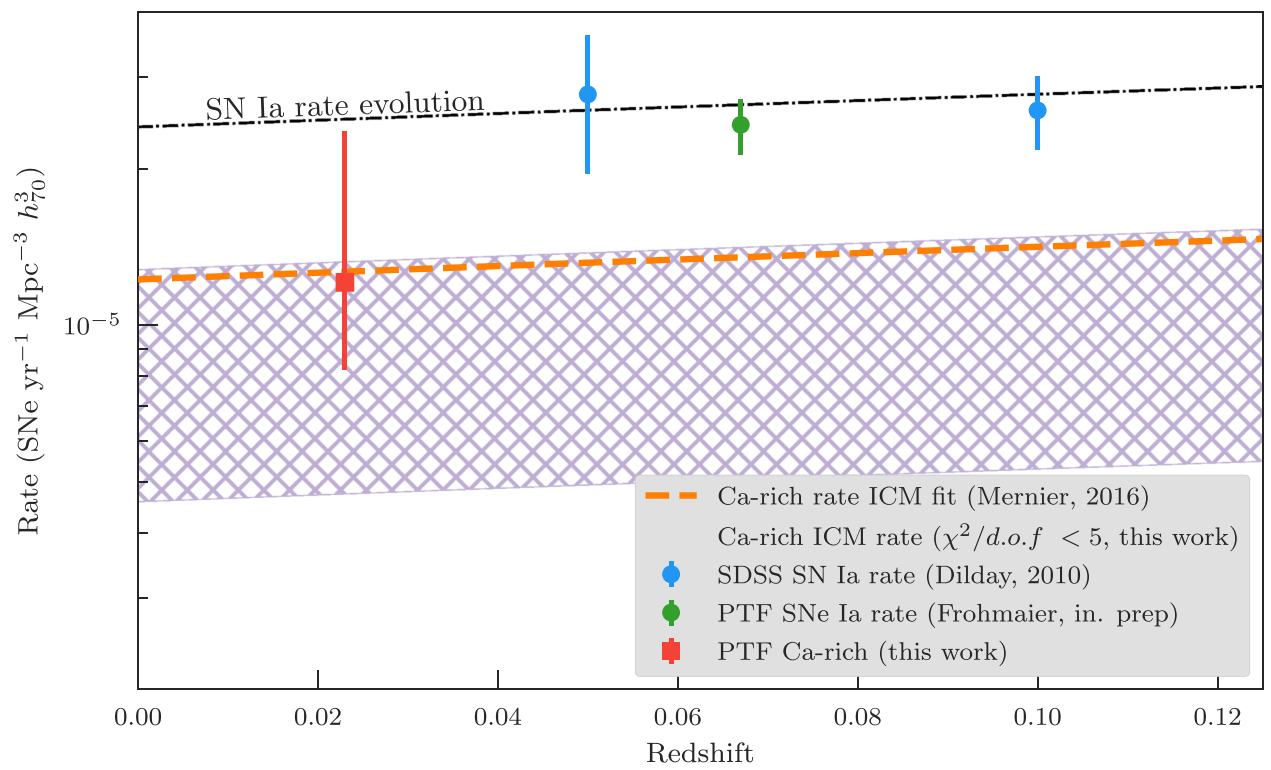

Figure 5. Volumetric rate of various thermonuclear SNe in the local universe. Our new Ca-rich measurement is shown as the red square. The blue circles show SN Ia rates from SDSS-SN (Dilday et al. 2010), and the green circle is from PTF (C. Frohmaier et al. 2018, in preparation). The purple hatched region shows the possible range of Ca-rich rates from our matching of different SN class elemental yields to ICM abundance observations. The dashed orange line shows the Ca-rich rate prediction by Mernier et al. (2016b) from their best fit to the ICM data.

galaxy centers; and (iii) we demonstrate that a non-negligible contribution from Ca-rich transients, compatible with our rate, can explain the observed ICM elemental abundances.

Attempts to unveil the intriguing nature of these transients will benefit significantly from upcoming high-cadence, widearea (ZTF) and deep (LSST) sky surveys, where a larger number of objects can be discovered. Key to this understanding will be early detections so that follow-up spectra and multicolor photometric observations can be obtained. Ultimately, studies on light-curve diversity and evolution will lead to tighter constraints on the explosion models and the asymptotic elemental yields for ICM abundance analyses. Of course, large samples of Ca-rich events will also improve the statistical uncertainties associated with the rate calculations and offer better insights into the host environments of these transients.

We thank F. Mernier for making the abunfit code publicly available and for the support we received in using it. We extend our gratitude to $\mathrm{K}$. Shen for providing us with the decayed yields of the sub- $M_{\mathrm{Ch}} \mathrm{SN}$ models. We also thank M. Kasliwal for useful comments on the manuscript prior to submission. Finally, we thank the organizers and participants of the Munich Institute for Astro- and Particle Physics (MIAPP) workshop "The Physics of Supernovae" for the stimulating discussions that started this work. M.S. acknowledges support from EU/FP7-ERC grant no. 615929. K.M. acknowledges support from the STFC through an Ernest Rutherford Fellowship. We acknowledge the use of the IRIDIS High Performance Computing Facility and associated support services at the University of Southampton in the completion of this work. P.N. acknowledges support from the DOE through DE-FOA-0001088, Analytical Modeling for Extreme-Scale Computing Environments. This research used resources of the National Energy Research Scientific Computing Center, a DOE Office of Science User Facility supported by the Office of Science of the U.S. Department of Energy under contract no. DE-AC02-05CH11231.

We thank the anonymous referee for useful comments.
Facility: PO:1.2m.

Software: astropy (Astropy Collaboration et al. 2013), astropy/photutils (Bradley et al. 2016), abunfit (Mernier et al. 2016b), Matplotlib (Hunter 2007), NumPy and SciPy (van der Walt et al. 2011), LMFIT (Newville et al. 2014).

\section{ORCID iDs}

Chris Frohmaier (1) https://orcid.org/0000-0001-9553-4723

Mark Sullivan (1) https://orcid.org/0000-0001-9053-4820

Kate Maguire (1) https://orcid.org/0000-0002-9770-3508

Peter Nugent 누 https://orcid.org/0000-0002-3389-0586

\section{References}

Astropy Collaboration, Robitaille, T. P., Tollerud, E. J., et al. 2013, A\&A, 558, A33

Bazin, G., Palanque-Delabrouille, N., Rich, J., et al. 2009, A\&A, 499, 653

Bellm, E. 2014, in The Third Hot-wiring the Transient Universe Workshop, ed. P. R. Wozniak et al. (Santa Fe, NM), 27

Bradley, L., Sipocz, B., Robitaille, T., et al. 2016, astropy/photutils, v0.3, Zenodo, doi:10.5281/zenodo.164986

Crocker, R. M., Ruiter, A. J., Seitenzahl, I. R., et al. 2017, NatAs, 1, 0135

de Plaa, J., Werner, N., Bleeker, J. A. M., et al. 2007, A\&A, 465, 345

Dilday, B., Smith, M., Bassett, B., et al. 2010, ApJ, 713, 1026

Filippenko, A. V., Li, W. D., Treffers, R. R., \& Modjaz, M. 2001, in ASP Conf. Ser. 246, IAU Coll. 183: Small Telescope Astronomy on Global Scales, ed. B. Paczynski, W.-P. Chen, \& C. Lemme, 121

Foley, R. J. 2015, MNRAS, 452, 2463

Frohmaier, C., Sullivan, M., Nugent, P. E., Goldstein, D. A., \& DeRose, J. 2017, ApJS, 230, 4

García-Berro, E., Badenes, C., Aznar-Siguán, G., \& Lorén-Aguilar, P. 2017, MNRAS, 468, 4815

Hunter, J. D. 2007, CSE, 9, 90

Kasliwal, M. M., Kulkarni, S. R., Gal-Yam, A., et al. 2012, ApJ, 755, 161

Laher, R. R., Masci, F. J., Groom, S., et al. 2017, arXiv:1708.01584

Lunnan, R., Kasliwal, M. M., Cao, Y., et al. 2017, ApJ, 836, 60

Lyman, J. D., Levan, A. J., Church, R. P., Davies, M. B., \& Tanvir, N. R. 2014, MNRAS, 444, 2157

Lyman, J. D., Levan, A. J., James, P. A., et al. 2016, MNRAS, 458, 1768

Mernier, F., de Plaa, J., Pinto, C., et al. 2016a, A\&A, 592, A157

Mernier, F., de Plaa, J., Pinto, C., et al. 2016b, A\&A, 595, A126 
Metzger, B. D. 2012, MNRAS, 419, 827

Mulchaey, J. S., Kasliwal, M. M., \& Kollmeier, J. A. 2014, ApJL, 780, L34

Newville, M., Stensitzki, T., Allen, D. B., \& Ingargiola, A. 2014, LMFIT: NonLinear Least-Square Minimization and Curve-Fitting for Python, Zenodo, doi: 10.5281 /zenodo. 11813

Nomoto, K., Kobayashi, C., \& Tominaga, N. 2013, ARA\&A, 51, 457

Perets, H. B., Gal-Yam, A., Mazzali, P. A., et al. 2010, Natur, 465, 322

Perrett, K., Sullivan, M., Conley, A., et al. 2012, AJ, 144, 59

Prajs, S., Sullivan, M., Smith, M., et al. 2017, MNRAS, 464, 3568

Rau, A., Kulkarni, S. R., Law, N. M., et al. 2009, PASP, 121, 1334

Rosswog, S., Kasen, D., Guillochon, J., \& Ramirez-Ruiz, E. 2009, ApJL, 705, L128
Seitenzahl, I. R., Ciaraldi-Schoolmann, F., Röpke, F. K., et al. 2013, MNRAS, 429, 1156

Sell, P. H., Maccarone, T. J., Kotak, R., Knigge, C., \& Sand, D. J. 2015, MNRAS, 450, 4198

Shen, K. J., Kasen, D., Miles, B. J., \& Townsley, D. M. 2017, arXiv:1706. 01898

Sullivan, M., Kasliwal, M. M., Nugent, P. E., et al. 2011, ApJ, 732, 118

Valenti, S., Yuan, F., Taubenberger, S., et al. 2014, MNRAS, 437, 1519

van der Walt, S., Colbert, S. C., \& Varoquaux, G. 2011, CSE, 13, 22

Waldman, R., Sauer, D., Livne, E., et al. 2011, ApJ, 738, 21

Yuan, F., Kobayashi, C., Schmidt, B. P., et al. 2013, MNRAS, 432, 1680

Zaritsky, D., Gonzalez, A. H., \& Zabludoff, A. I. 2004, ApJL, 613, L93 